\title{
Prototype of Self-Sensing Magnetic Bearing for Liquid Nitrogen Pump
}

\author{
Seiji Eguchi Non-member (Kyushu Institute of Technology) \\ Mochimitsu Komori Member (Kyushu Institute of Technology) \\ Taro Okuhata Non-member (Kyushu Institute of Technology)
}

Keywords: self-sensing, magnetic bearing, liquid nitrogen pump

\section{Introduction}

There are a lot of studies of actuators which work in extremely low temperature such as liquid nitrogen and liquid hydrogen. If such magnetic bearings were realized, they would be free from their maintenances. Thus, we propose a self-sensing magnetic bearing able to work in extremely low temperature.

\section{Structure}

We propose a pump with self-sensing magnetic bearings as shown in Fig. 1. The rotor is supported by five pairs of self-sensing magnetic bearings. Four pairs of them support the rotor in the radial direction, and a pair of them support in the thrust direction. An impeller and a motor are installed in the pump.

\section{Experimental Setup}

Fig. 2 shows an experimental setup. The rotor is supported by a hinge in the upper part, and it is possible to move freely only in the $\mathrm{X}$ direction. Since the magnetic bearing is vertically installed, the effect of gravity is neglected. The sensing coil and the control coil are installed in the self-sensing magnetic bearing.

\section{Self-Sensing}

Relationship between inductance and displacement from the center is evaluated. The measurement was performed in the air and in liquid nitrogen. In each case, the inductances of the upper and lower coils are proportional to the displacement. The inductance in liquid nitrogen is a little smaller than that in the air. This is because the magnetic characteristic of the core material changes in extremely low temperature.

\section{Results and Discussion}

Impulse responses for the self-sensing magnetic bearing in the air were performed. The result shows that vibrations are observed due to the applied impulse. The displacement by using an eddy current sensor is almost equal to the displacement by using the self-sensing

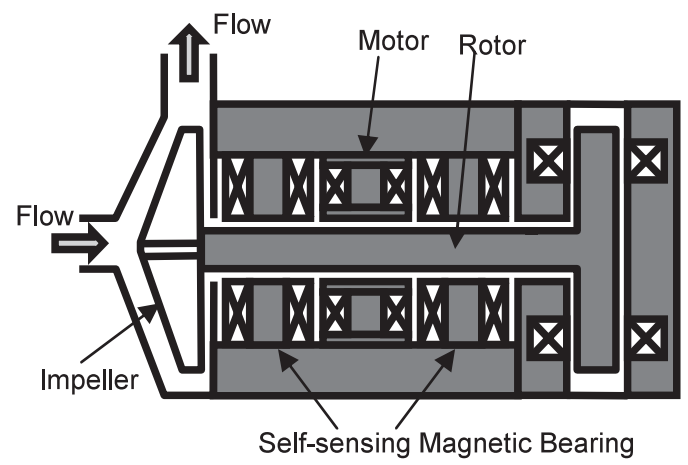

Fig. 1. Structure of a liquid nitrogen pump sensor. The vibrations of the rotor disappear within $0.5 \mathrm{~s}$ just after the impulse is applied.

Impulse responses for the self-sensing magnetic bearing in liquid nitrogen were also performed as shown in Fig. 3. The result shows that vibrations of the rotor are observed due to the impulse. The vibrations of the rotor disappear within $0.2 \mathrm{~s}$ just after the impulse is applied. The vibrations of the rotor in liquid nitrogen are a little smaller than the vibrations in the air.

\section{Conclusions}

We proposed a pump with self-sensing magnetic bearings which works in extremely low temperature. Self-sensing experiments were performed in the air and in liquid nitrogen. The vibrations of the rotor are a little smaller and damping is considerably larger than those in the air.

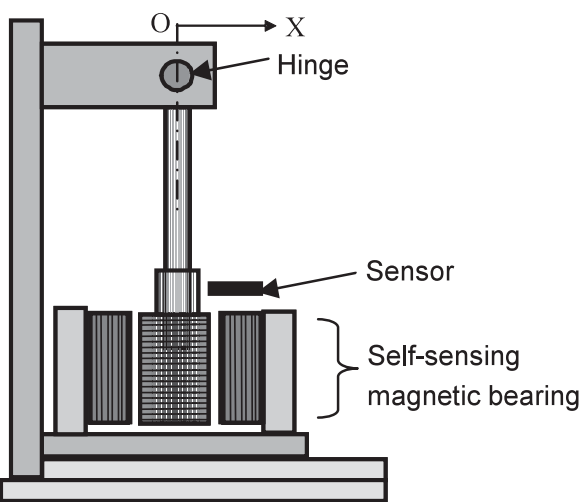

Fig. 2. Experimental setup of self-sensing magnetic bearing

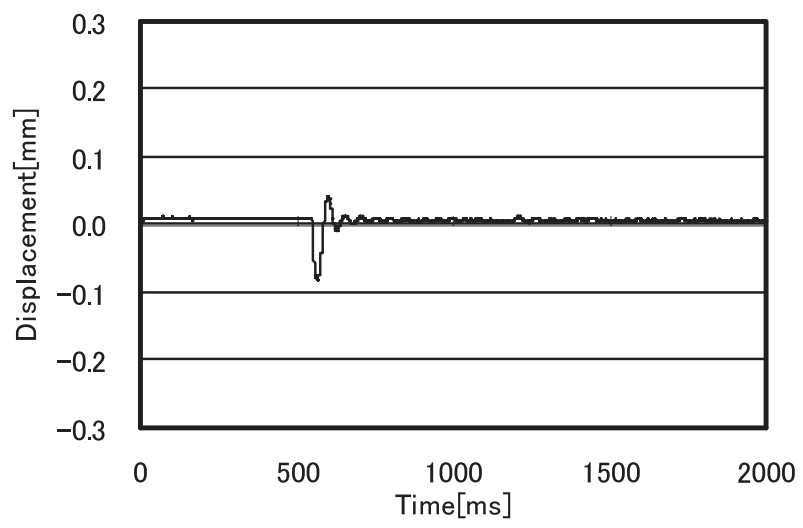

Fig. 3. Self-sensing displacement (in liquid nitrogen) 


\title{
Prototype of Self-Sensing Magnetic Bearing for Liquid Nitrogen Pump
}

\author{
Seiji Eguchi* \\ Mochimitsu Komori* \\ Non-member \\ Taro Okuhata* \\ Member \\ Non-member
}

\begin{abstract}
Recently, pumps used in extremely low temperature such as $77 \mathrm{~K}$ are found to be necessary. They are expected to use for rocket engines and hydrogen stations for fueled vehicles. Generally, conventional magnetic bearings do not work in the extremely low temperature. Therefore, we have studied magnitic bearings for these pumps. Self-sensing technique is tried to apply to magnetic bearings. If self-sensing magnetic bearings were made, we could apply the self-sensing magnetic bearing to liquid nitrogen pumps. In this paper, we propose a prototype self-sensing magnetic bearing and study the static and dynamic characteristics. The dynamic characteristics in the air and in liquid nitrogen are also discussed.
\end{abstract}

Keywords: self-sensing, magnetic bearing, liquid nitrogen pump

\section{Introduction}

Recently, magnetic bearings are used in various fields because of its advantage of maintenance. And various types of magnetic bearings have been proposed ${ }^{(1)-(3)}$. On the other hand, there are very few studies of actuators which work in extremely low temperature such as liquid nitrogen and liquid hydrogen except for superconducting actuators ${ }^{(4)(5)}$. If such magnetic bearings without mechanical contacts were realized, they would be free from their maintenances. Generally a magnetic bearing needs some displacement sensors for sensing the displacement of the rotor. However the conventional magnetic bearings do not work in extremely low temperature. Generally, displacement sensors and electromagnets can't be arranged in the same place because electromagnets effect on displacement sensors. Thus, we propose a self-sensing magnetic bearing able to work in extremely low temperature. In this study, we made a prototype of selfsensing magnetic bearing and studied the characteristics.

\section{Structure of a Liquid Nitrogen Pump}

We propose a pump with self-sensing magnetic bearings able to work in extremely low temperature. Fig. 1 shows the structure of the liquid nitrogen pump with self-sensing magnetic bearings. The temperature of liquid nitrogen is $77 \mathrm{~K}$. The rotor is supported by five pairs of self-sensing magnetic bearings without mechanical contacts. Four pairs of them support the rotor in the radial direction, and a pair of them support in the thrust direction. An impeller and a motor are installed in the pump. Liquid nitrogen flows into the pump from the left hand side when the motor works. Then, liquid nitrogen flows out from the upper part of the pump.

\section{Principle of Self-sensing}

In this study, we adopted inductance type self-sensing techunique. Fig. 2 shows a basic circuit of the inductance

\footnotetext{
* Kyusyu Institute of Technology

1-1, Sensui-cho, Tobata-ku, Kitakyushu 804-8550
}

type sensor. $L_{1}$ shows the inductance of upper coil, and $L_{2}$ the inductance of lower coil. $v_{0}$ is a voltage applied to the coils $L_{1}$ and $L_{2}$. If the rotor changes the position, each of the inductances changes depending on the displacement of the rotor. Thus, each of the inductances is represented as equations (1) and (2), where $L_{c o n}$ is constant, $L^{\prime}$ is the difference corresponding to the displacement.

$$
\begin{aligned}
& L_{1}=\frac{L_{c o n}}{2}+L^{\prime} d . \\
& L_{2}=\frac{L_{c o n}}{2}-L^{\prime} d .
\end{aligned}
$$

Middle point voltage $v_{1}$ is a voltage for the inductance of the

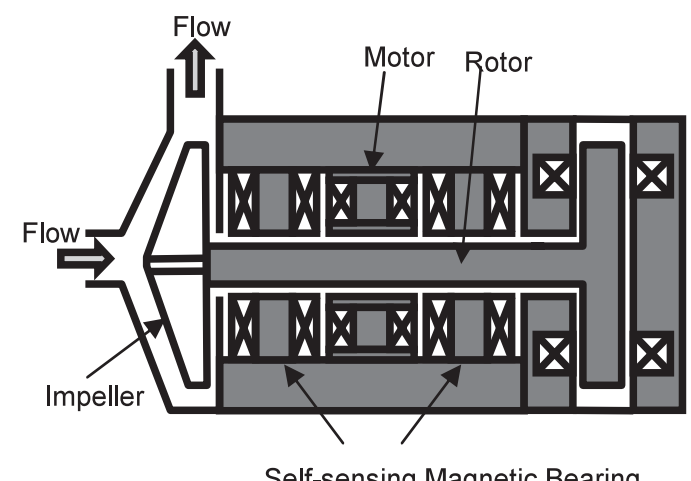

Fig. 1. Structure of a liquid nitrogen pump

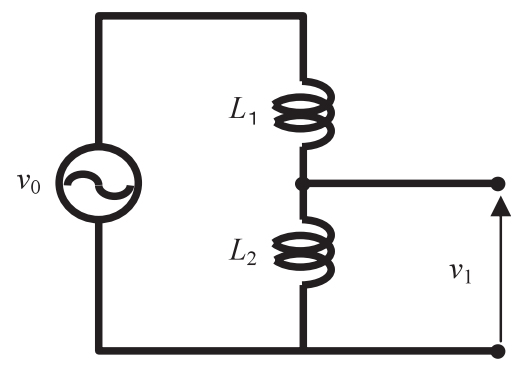

Fig. 2. Inductance sensor 


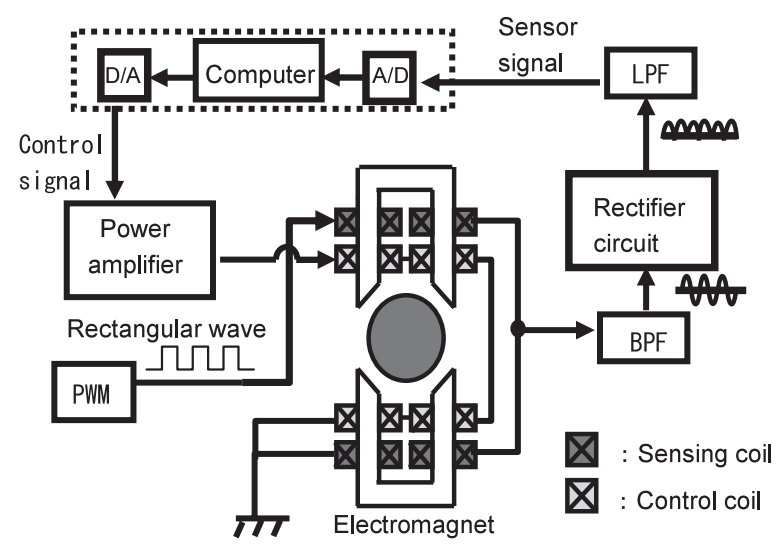

Fig. 3. Self-sensing magnetic bearing system

lower coil. It is represented as

$$
v_{1}=L_{2} \frac{d i_{0}}{d t}
$$

where $i_{0}$ is a current that flows into the coil. Substituting equations (1) and (2) to equation (3), we have

$$
v_{1}=\frac{1}{2}\left(1-\frac{2 L^{\prime}}{L_{c o n}} d\right) v_{0}
$$

According to equation (4), middle point voltage $v_{1}$ changes depending on the displacement of the rotor. By detecting middle point voltage $v_{1}$, it is possible to evaluate the displacement of the rotor.

\section{Self-sensing System}

Fig. 3 shows a block diagram of the self-sensing magnetic bearing system. Two coils for sensing and for control are installed in each of the upper and lower electromagnet. Each of these coils is connected in series. Bias current is given to the sensing coil using PWM signal. The voltage between upper and lower coil changes depending on displacement of the rotor. The middle point voltage passes through the band pass filter (BPF) and the carrier element (sensing signal) is detected. After passing through the rectification circuit and the low pass filter (LPF), the sensing signal goes into the computer through the A/D converter. The control signal is amplified and applied to the control coil. In the upper electromagnet, the sensing coil and the control coil are connected in the same direction. In the lower electromagnet, the sensing coil and the control coil are connected in the opposite direction. Thus, the attractive force is produced in the vertical direction.

\section{Attractive Force}

Fig. 4 shows the principle of attractive forces. In Fig. 4 , the solid lines show the magnetic flux density $\left(B_{S}\right)$ by sensing coil, the broken lines show the magnetic flux density $\left(B_{c}\right)$ by control coil. We regard the upper and lower electromagnet as a magnetic circuit. And the magnetic flux density of upper coil is $B_{1}$, and the magnetic flux density of lower coil is $B_{2}$. Magnetic flux densities $B_{1}$ and $B_{2}$ are represented as equation (5) and (6), respectively.

$$
\begin{aligned}
& B_{1}=B_{s}+B_{c} \\
& B_{2}=B_{s}-B_{c}
\end{aligned}
$$

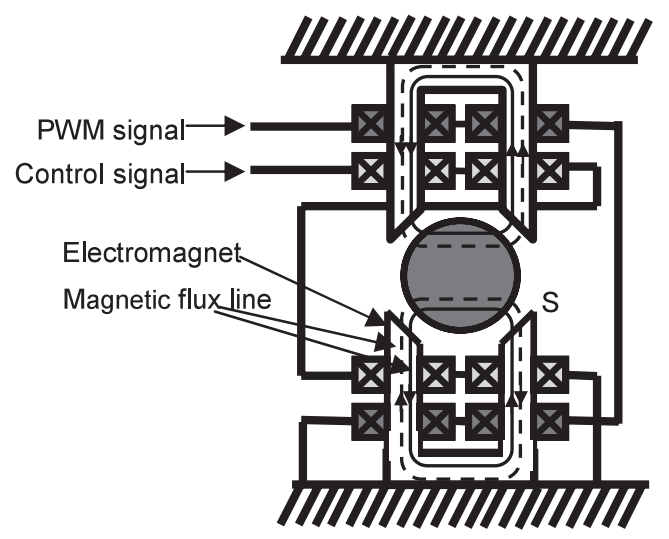

Fig. 4. Principle of attractive forces

The direction of the control flux changes corresponding to the control current. Therefore, the attractive force $f$ for the rotor can be controlled. Generally, the attractive force $f$ for an electromagnet is represented by equation (7),

$$
f=\frac{S B^{2}}{\mu_{0}},
$$

where $S$ is the cross-sectional area of electromagnets. Thus, total attractive force $F$ for upper and lower electromagnets is given by

$$
F=\frac{S}{\mu_{0}}\left(B_{1}^{2}-B_{2}^{2}\right) .
$$

When equations (5) and (6) are substituted to equation (8), equation (8) is written as

$$
F=4 \frac{S}{\mu_{0}} B_{s} B_{c} .
$$

Since magnetic flux density $B_{s}$ of the sensing coil is constant, equation (9) can be represented as

$$
F=\alpha B_{c}
$$

where

$$
\alpha=4 \frac{S}{\mu_{0}} B_{s}
$$

The attractive force $F$ of the electromagnet is proportional to the magnetic flux density $B_{s}$ of the control coil.

\section{Experimental Setup}

Fig. 5 shows the experimental setup used in this study. The rotor is supported by a hinge in the upper part, and it is possible to move freely only in the X direction. Since the magnetic bearing is vertically installed, the effect of gravity is neglected. The sensing coil and the control coil are installed in the self-sensing magnetic bearing. A displacement sensor is used to detect the correct displacement.

\section{Self-Sensing}

Fig. 6 shows the relationship between inductance and displacement from the center. The measurement was performed in the air and in liquid nitrogen. In each case, the inductances of the upper coil and the lower coil are in proportion to the displacement. The inductance in liquid nitrogen is a 


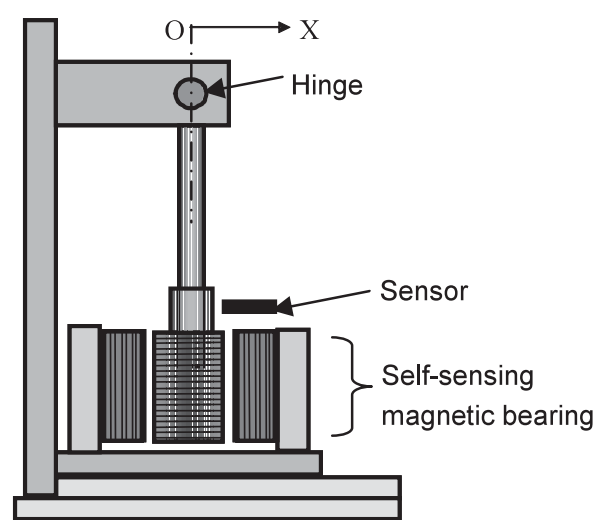

Fig. 5. Experimental setup of self-sensing magnetic bearing
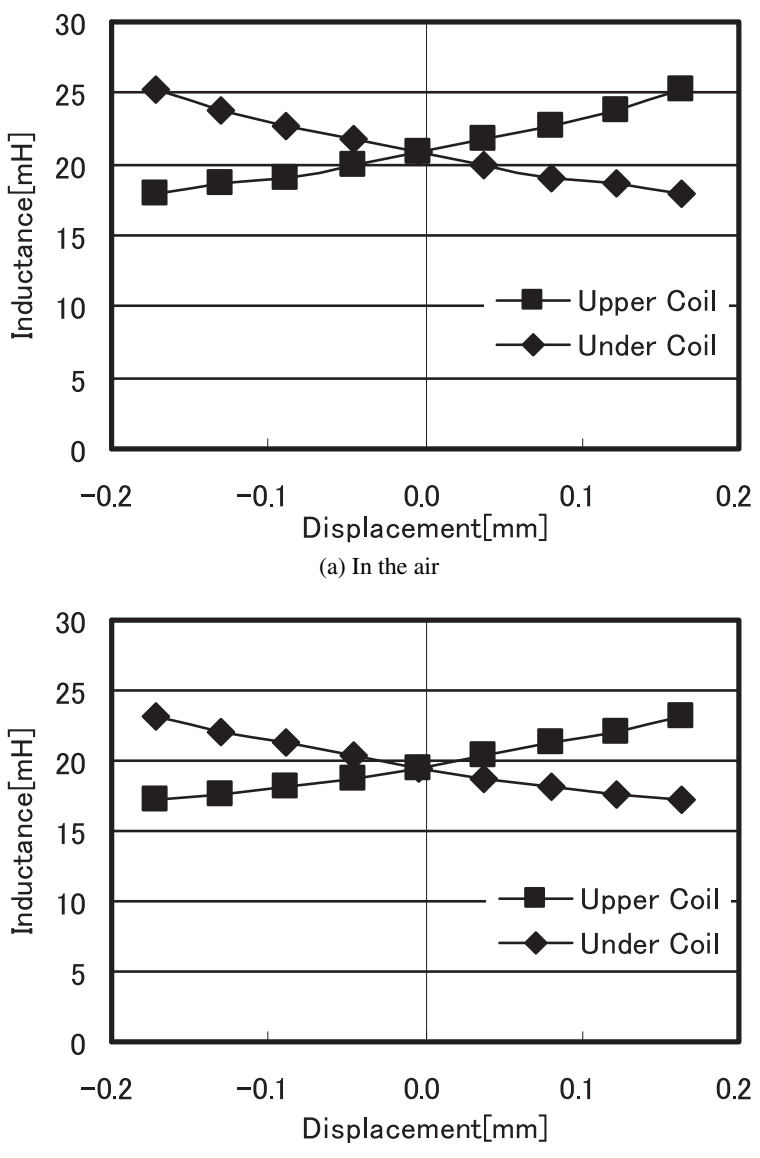

(b) In liquid nitrogen

Fig. 6. Relationships between displacement and inductance (a) in the air and (b) in liquid nitrogen

little smaller than that in the air. This is because the magnetic characteristic of the core material changes in extremely low temperature. However, this is not so important because the inductance in liquid nitrogen is also proportional to the displacement of the rotor.

First, we set the rotor in the center of the magnetic bearing and measure the middle point voltage $v_{1}$ when the rotor moves from $-0.2 \mathrm{~mm}$ to $0.2 \mathrm{~mm}$. Fig. 7 shows the relationships between middle point voltage and displacement for (a) $5 \mathrm{kHz}$, (b) $8 \mathrm{kHz}$ and (c) $11 \mathrm{kHz}$. In each figure, the control currents from $-0.5 \mathrm{~A}$ to $0.5 \mathrm{~A}$ are applied to the electromagnet. Each of the middle point voltages changes in propor-
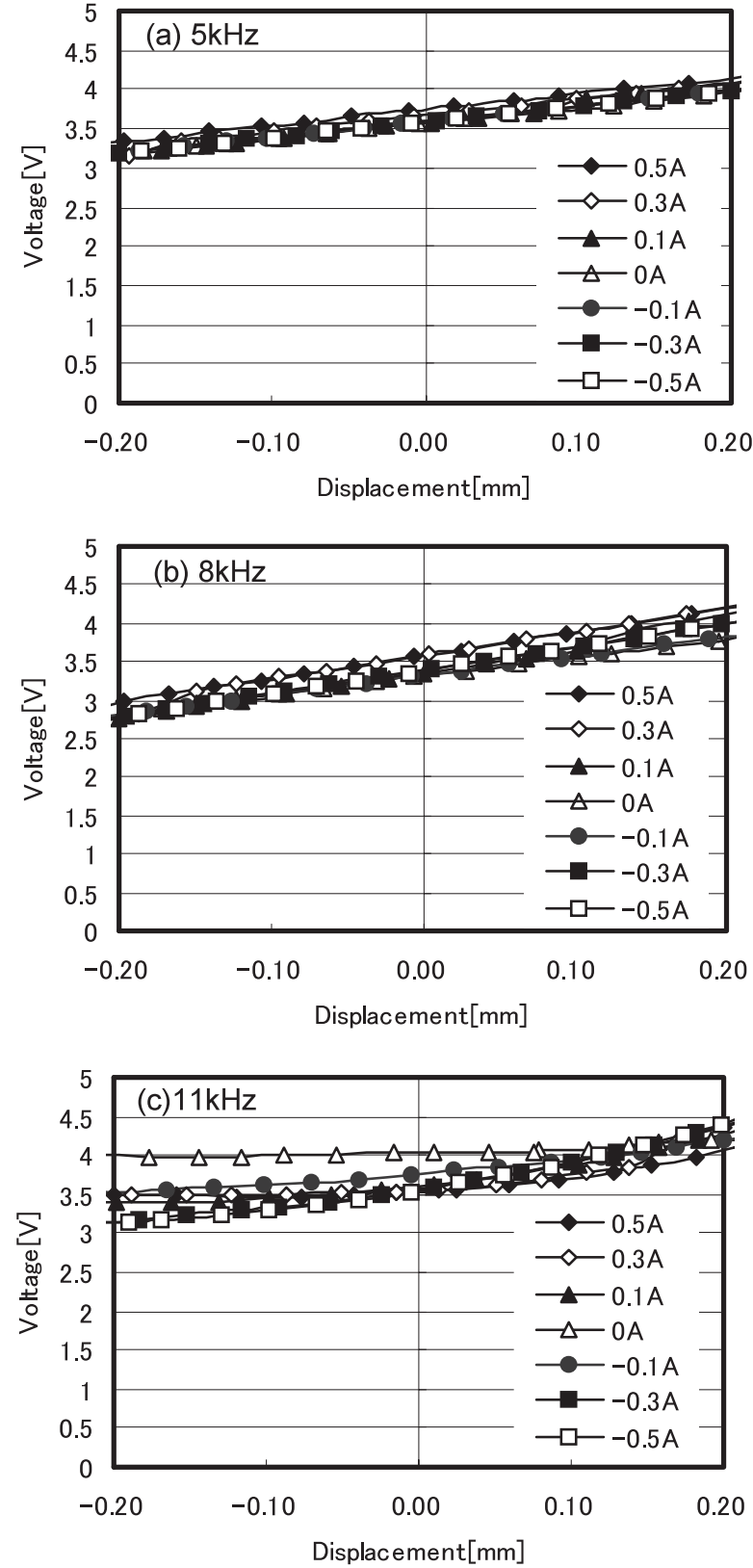

Fig. 7. Relationships between middle point voltage and control current for (a) $5 \mathrm{kHz}$, (b) $8 \mathrm{kHz}$, and (c) $11 \mathrm{kHz}$

tional to the displacement. However, each voltage has a little difference depending on the control current from $-0.5 \mathrm{~A}$ to $0.5 \mathrm{~A}$. Moreover, the difference of middle point voltage at the same displacement becomes large with increasing frequency from $5 \mathrm{kHz}$ to $11 \mathrm{kHz}$. The result shows that the lower frequency $5 \mathrm{kHz}$ is better than the higher frequency $11 \mathrm{kHz}$. However, lower frequencies effect on the control signal. Thus, in the experiment we adopted $5 \mathrm{kHz}$ for the reason mentioned above.

\section{Results and Discussion}

In order to prove the self-senisng technique, we had some experiments for the self-sensing magnetic bearing in the air and in liquid nitrogen. First, impulse responses for the selfsensing magnetic bearing in the air were performed. The impulses were applied by using a small alminium hummer. 


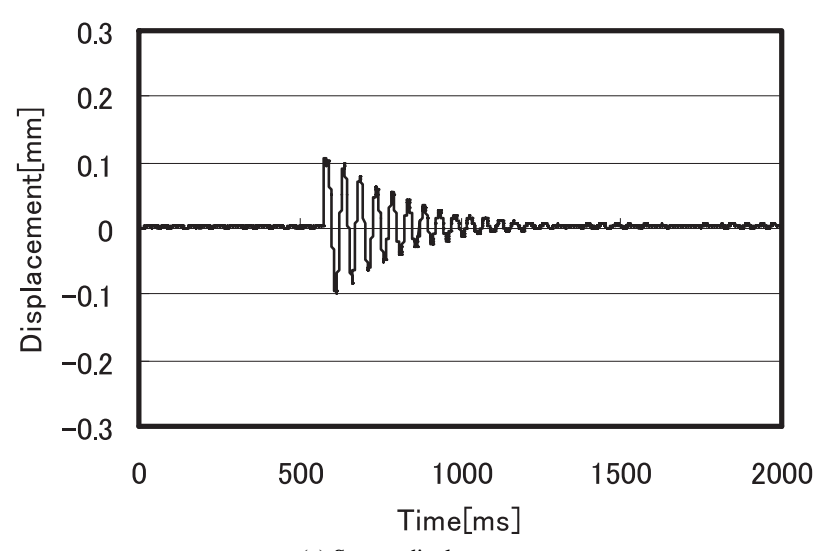

(a) Sensor displacement

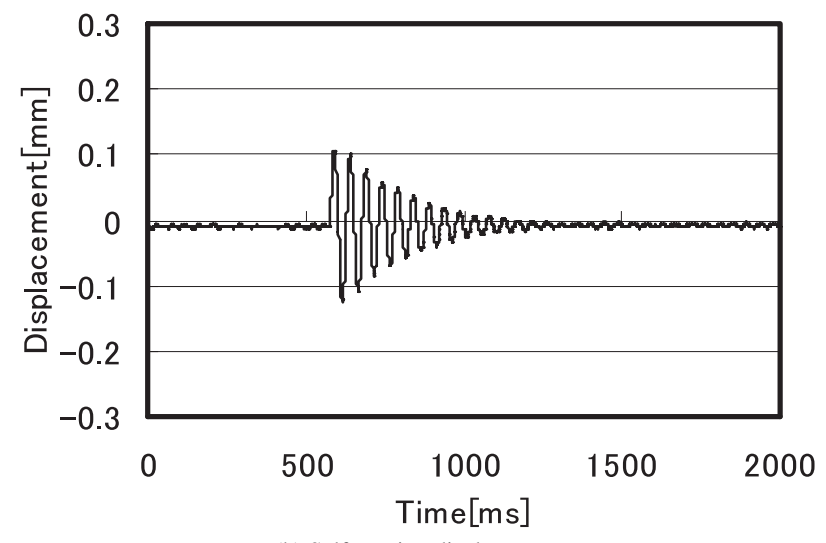

(b) Self-sensing displacement

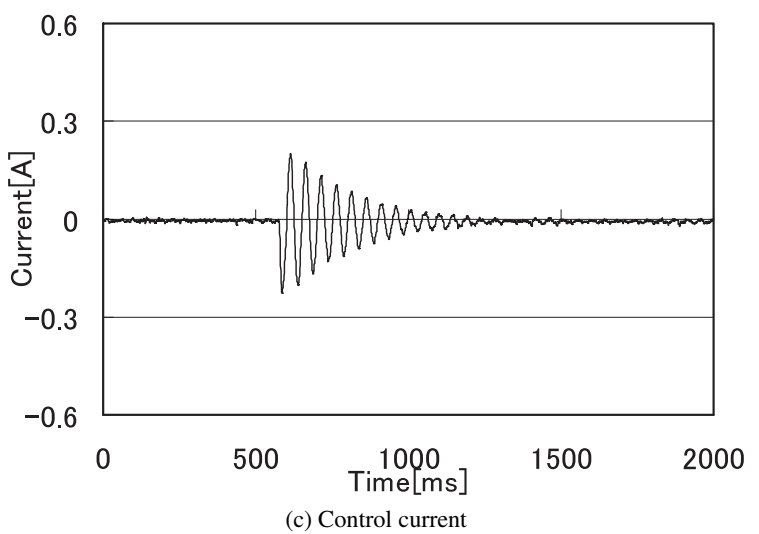

Fig. 8. Self-sensing displacement (in the air)

Fig. 8 shows the results of the impulse response, showing (a) the displacement by using an eddy current displacement sensor, (b) the displacement by using the self-sensing technique, and (c) the control current to electromagnet. These results are obtained at the same time. Figs. 8(a) and (b) show that vibrations are observed due to the applied impulse. The displacement in Fig. 8 (a) is almost equal to the displacement in Fig. 8(b). The control current in Fig. 8(c) corresponds to the displacement in Fig. 8(b). The vibrations of the rotor disappear within $0.5 \mathrm{~s}$ just after the impulse is applied.

Impulse responses for the self-sensing magnetic bearing in liquid nitrogen were performed. Fig. 9 shows the results of the impulse response, showing (a) the displacement by using the self-sensing technique and (b) the control current to electromagnet. The displacement by using an eddy current

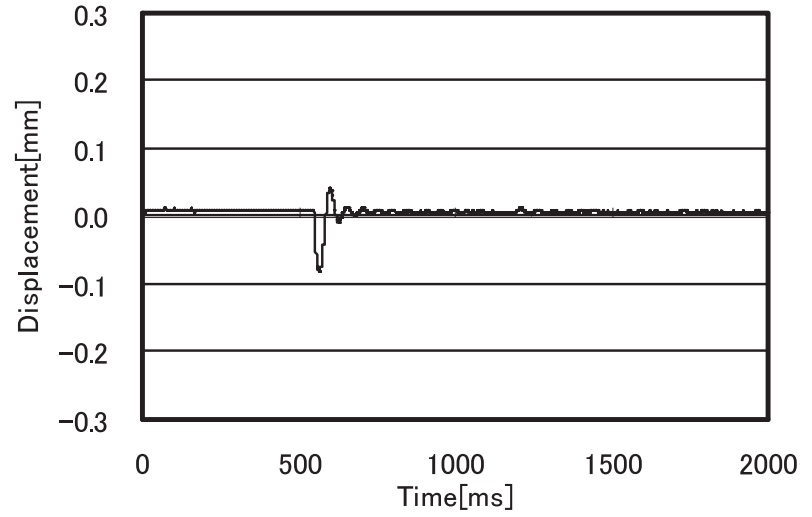

(a) Self-sensing displacement

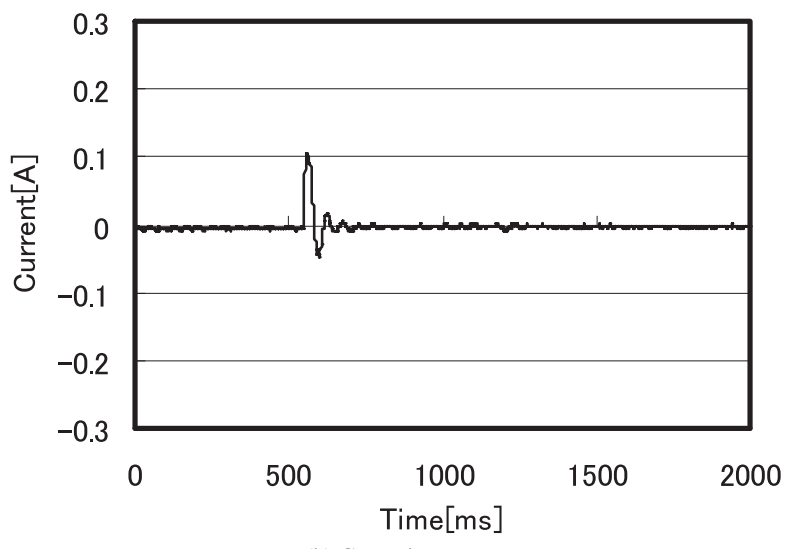

(b) Control current

Fig. 9. Self-sensing displacement (in liquid nitrogen)

sensor is not shown in Fig. 9, because the sensor dose not work in liquid nitrogen. Fig. 9(a) shows that vibrations of the rotor are observed due to the impulse. The control current in Fig. 9(b) corresponds to the displacement in Fig. 9(a). The vibrations of the rotor disappear within $0.2 \mathrm{~s}$ just after the impulse is applied. The vibrations of the rotor in liquid nitrogen (Fig. 9(a)) are a little smaller than the vibrations in Fig. 8(b). The damping in Fig. 9(a) is considerably larger than that (Fig. 8(b)) in the air. This is because liquid nitrogen increases the damping.

In this paper, we made a prototype of self-sensing magnetic bearing able to work in extremely low temperature. Thus, the details of the self-sensing magnetic bearing such as frequency dependence and time constant of the sensor coils, and linearity of the sensing signal are not discussed. Detailed studies have to be done to clarify these problems.

\section{Conclusions}

We proposed a pump with self-sensing magnetic bearings which works in extremely low temperature. Self-sensing experiments were performed in the air and in liquid nitrogen. It was confirmed that the sensor displacement and the selfsensing displacement were almost the same and the vibrations were well suppressed. Comparing the dynamics in the air with those in liquid nitrogen, the vibrations of the rotor are a little smaller and damping is considerably larger than those in the air.

(Manuscript received Nov. 28, 2005,

revised April 3, 2006) 


\section{References}

( 1 ) O. Matsuda, Y. Okada, and J. Tani: "Self-sensing Magnetic Bearing using the Principle of Differential Transformer", JSME Journal (C), Vol.63, pp.14411447 (1997)

( 2 ) K. Ebara, T. Nobe, A. Chiba, and T. Fukao: "A Method of Rotor Position and Motor Flux Detection with Search Coils in Bearingless Motors", The 14th Symposium on Electromagnetcs and Dynamics, pp.397-402, Okayama (2002-5) (in Japanese)

( 3 ) T. Higuchi and T. Mizuno: "Control system design for totally active DC-type magnetic bearings", Trans. of the Society of Instrument and Control Engineers, Vol.18, pp.507-513 (1982)

( 4 ) M. Komori and K. Uchino: "Development of a liquid nitrogen pump using superconducting bulk motor", IEEE Trans. AS, Vol.14, pp.1659-1662 (2004)

( 5 ) H.-J. Jung, T. Nakamura, I. Muta, and T. Hoshino: "Characteristics of axialtype HTS motor under different temperature conditions", IEEE Trans. AS, Vol.13, pp.2201-2205 (2003)

Seiji Eguchi (Non-member) received Bachelor and Master degrees

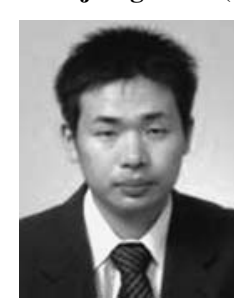
from Kyushu Institute of technology in 2003 and 2005, respectively. He was a Master course student, Dept. of Applied Science for Integrated System Engineering, Kyushu Institute of Technology. His main research interest is in self-sensing magnetic levitation.
Mochimitsu Komori (Member) received MS degree from Osaka Uni-

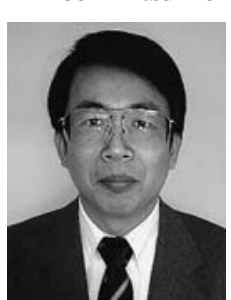
versity in 1984 and Ph.D. degree from Kyushu University. He is now a professor at the Dept. of Applied Science for Integrated System Engineering, Kyushu Institute of Technology. His main research interest is in superconducting levitation, magnetic levitation, passive levitation, micro levitation and their engineering applications.

Taro Okuhata (Non-member) received Bachelor degree from Kyushu

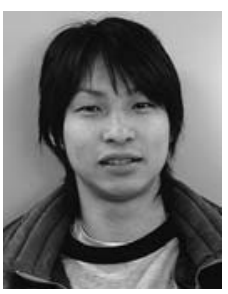
Institute of technology in 2005. He is a Master course student at Komori's Laboratory, Kyushu Institute of Technology. His main research interest is in selfsensing magnetic levitation. 